\title{
SYNTHESIS OF AZASUGARS THROUGH A
}

\section{PROLINE-CATALYZED REACTION}

\section{Supporting Information}

Félix Calderón, Elisa G. Doyagüez and Alfonso Fernández-Mayoralas*

Instituto de Química Orgánica General, CSIC, c/ Juan de la Cierva 3, 28006 Madrid, Spain.

mayoralas@iqog.csic.es

\section{Table of contents}

1.- General methods (S2)

2.- Compound list (S3-S4)

3.- Experimental procedures for compounds $\mathbf{5}, \mathbf{6}, \mathbf{7}, \mathbf{8}, \mathbf{3}$, ent-5, ent-6, ent-7, ent-8 and ent-3 (S5-S7)

4.- Selected ${ }^{1} \mathrm{H},{ }^{13} \mathrm{C}-\mathrm{NMR}$ and key $2 \mathrm{D}-\mathrm{NMR}$ spectra:

- S8: 9a

- S10: 9b

- S12: 10a

- S14: 10b

- S15: 11

- $\mathrm{S} 16: \mathbf{1 3 a}+\mathbf{1 3 b}$

- S17: 15

- S19: 18 


\section{General methods}

Chemicals were purchased puriss p.A. from commercial suppliers or purified by standard techniques. Thin-layer chromatography (TLC) was performed on aluminium sheets $60 \mathrm{~F}_{254}$ silica gel and compounds were visualized by irradiation with UV light and/or by treatment with a solution of $\mathrm{Ce}_{2} \mathrm{MoO}_{4}$ followed by heating. Flash chromatography was performed using thick walled columns, employing silica gel (0.040-0.063 nm). NMR $\left({ }^{1} \mathrm{H},{ }^{13} \mathrm{CNMR}\right)$ spectra were recorded on a $300 \mathrm{MHz}$ spectrometer, using $\mathrm{CDCl}_{3}$ as solvent at room temperature. Chemical shift values are reported in parts per million $(\delta)$ relative to tetramethylsilane (TMS) in ${ }^{1} \mathrm{H}$ and $\mathrm{CDCl}_{3}(\delta=77.0)$ in ${ }^{13} \mathrm{C}$ NMR. Coupling constant ( $J$ values) are reported in hertz $(\mathrm{Hz})$, and spin multiplicities are indicated by the following symbol: s (singlet), $d$ (doublet), $t$ (triplet), q (quartet), $m$ (multiplet). 


\section{Compound List}<smiles>CC1(C)OCC(=O)CO1</smiles>

1<smiles>CC(=O)CO</smiles>

2<smiles>NC1COC(c2ccccc2)OC1C=O</smiles>

ent-3<smiles>CCOC(=O)C(O)[C@@H](O)C(=O)OCC</smiles>

4<smiles>CCOC(=O)C(O)[C@@H](O)C(=O)OCC</smiles>

ent-4

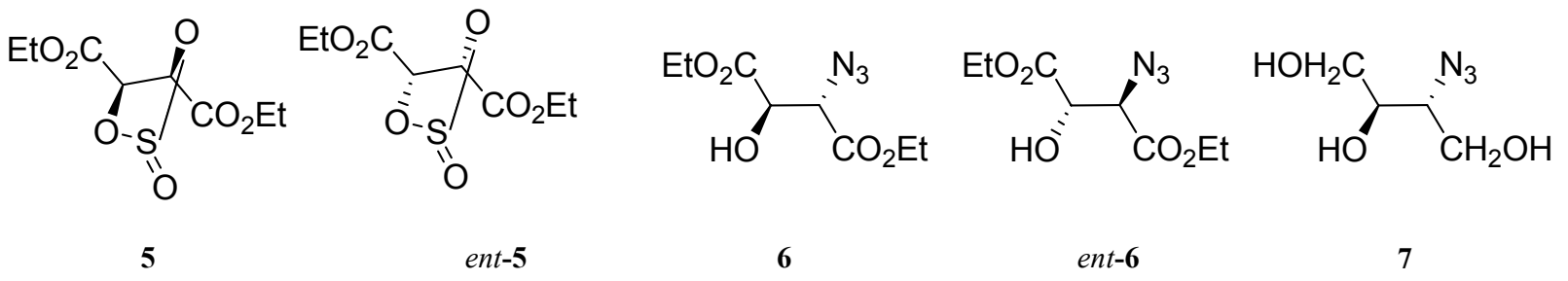<smiles>N[C@H](CO)[C@@H](O)CO</smiles>

ent-7<smiles>N#C[C@@H]1COC(c2ccccc2)OC1CO</smiles><smiles>N#CC(CCO)COC(O)c1ccccc1</smiles><smiles>CC1(C)OCC(=O)[C@H]([C@H](O)[C@H]2OC(c3ccccc3)OC[C@H]2N)O1</smiles>

9a<smiles>CC1(C)OCC(=O)[C@H]([C@H](O)[C@H]2OC(c3ccccc3)OC[C@H]2N)O1</smiles>

ent-9a<smiles>CC1(C)OCC(=O)[C@@H]([C@H](O)[C@H]2OC(c3ccccc3)OC[C@H]2N)O1</smiles><smiles>CC1(C)OCC(=O)C([C@H](O)[C@H]2OC(c3ccccc3)OC[C@H]2N)O1</smiles><smiles>CC(=O)[C@H](O)[C@H](O)[C@H]1OC(c2ccccc2)OC[C@H]1N</smiles><smiles>CC(=O)[C@H](O)[C@H](O)C1OC(c2ccccc2)OC[C@H]1N</smiles>

9b

ent-9b

10a

ent-10a<smiles>CC(=O)[C@H](O)[C@H](O)[C@H]1OC(c2ccccc2)OC[C@H]1N</smiles><smiles>CC(=O)C(O)[C@H](O)[C@H]1OC(c2ccccc2)OC[C@H]1N</smiles><smiles>OC[C@H]1N[C@H](CO)[C@@H](O)[C@H](O)[C@H]1O</smiles><smiles>OC[C@H]1N[C@H](CO)[C@@H](O)[C@H](O)[C@H]1O</smiles>

$10 \mathrm{~b}$

ent-10b

11

ent-11<smiles>OC[C@H]1N[C@H](CO)[C@@H](O)[C@H](O)C1O</smiles>

$12 \mathrm{a}$<smiles>OC[C@H]1N[C@H](CO)[C@@H](O)[C@H](O)[C@H]1O</smiles>

$12 b$<smiles>OC[C@H]1N[C@H](CO)C(O)C(O)C1O</smiles>

ent-12a<smiles>OC[C@H]1N[C@H](CO)[C@H](O)[C@@H](O)C1O</smiles>

ent-12b 
<smiles>C[C@@H]1N[C@H](CO)[C@@H](O)[C@H](O)[C@H]1O</smiles>

$13 a$<smiles>C[C@H]1N[C@H](CO)[C@@H](O)[C@H](O)[C@H]1O</smiles>

13b<smiles>C[C@H]1N[C@H](CO)[C@@H](O)[C@H](O)[C@H]1O</smiles>

ent-13a<smiles>C[C@@H]1N[C@H](CO)[C@@H](O)[C@H](O)[C@H]1O</smiles>

ent-13b<smiles>C[C@H]1N[C@H](CO)[C@@H](O)[C@H](O)[C@H]1O</smiles>

$14 a$<smiles>C[C@@H]1N[C@H](CO)[C@@H](O)[C@H](O)[C@H]1O</smiles>

14b<smiles>C[C@@H]1N[C@H](CO)[C@@H](O)[C@H](O)[C@H]1O</smiles>

ent-14a<smiles>C[C@@H]1N[C@H](CO)[C@@H](O)[C@H](O)[C@H]1O</smiles>

ent-14b<smiles>CC1(C)OC[C@H]2N[C@@H]3CO[C@H](c4ccccc4)O[C@H]3[C@H](O)[C@@H]2O1</smiles>

15<smiles>C[C@H]1N[C@@H]2CO[C@@H](c3ccccc3)O[C@H]2[C@H](O)[C@@H]1O</smiles>

18<smiles>C[C@H]1N[C@@H]2CO[C@@H](c3ccccc3)O[C@H]2[C@H](O)[C@@H]1O</smiles>

21<smiles>CC(=O)C1OC(C)(C)OC1[C@H]1OC(c2ccccc2)OC[C@H]1N</smiles>

24<smiles>CC1(C)OC[C@H]2N[C@@H]3CO[C@H](c4ccccc4)O[C@@H]3[C@H](O)[C@H]2O1</smiles>

16<smiles>C[C@H]1N[C@@H]2CO[C@@H](c3ccccc3)O[C@H]2[C@H](O)[C@@H]1O</smiles>

19<smiles>CC(=O)O[C@@H](C)[C@@H](OC(C)=O)[C@H]1OC(c2ccccc2)OC[C@H]1N</smiles>

22<smiles>[Y10]O[C@@H](C(C)=O)[C@@H](O[14CH3])[C@H]1OC(c2ccccc2)OC[C@H]1N</smiles>

25<smiles>CC1(C)OC[C@H]2N[C@@H]3CO[C@H](c4ccccc4)O[C@@H]3[C@H](O)[C@H]2O1</smiles>

17<smiles>C[C@H]1N[C@@H]2CO[C@@H](c3ccccc3)O[C@H]2[C@H](O)[C@@H]1O</smiles>

20<smiles>CC(=O)OC1[C@H](C)N[C@@H]2CO[C@@H](c3ccccc3)O[C@@H]2[C@H]1OC(C)=O</smiles>

23 


\section{Experimental procedures for compounds 5, 6, 7, 8, 3, ent-5, ent-6, ent-7, ent-8 and ent-3.}

Diethyl (2R, 3R)-2,3-O-sulfinyl-tartrate (5). Thionyl chloride (2.12 mL, $29.08 \mathrm{mmol})$ was added dropwise to a stirred solution of diethyl $(R, R)$ tartrate (4) (3 g, $14.54 \mathrm{mmol})$ and anhydrous triethylamine $(4.7 \mathrm{~mL}, 30 \mathrm{mmol})$ in dry $\mathrm{CH}_{2} \mathrm{Cl}_{2}\left(30^{1} \mathrm{~mL}\right)$ at $0^{\circ} \mathrm{C}$. The temperature was allowed to gradually raise to room temperature over $2 \mathrm{~h}$, then $\mathrm{CH}_{2} \mathrm{Cl}_{2}(20 \mathrm{~mL})$ and $\mathrm{NaCl}$ (sat) $(40 \mathrm{~mL})$ was added to the reaction mixture. The layers were separated and the aqueous phase was extracted thoroughly with $\mathrm{CH}_{2} \mathrm{Cl}_{2}$. The combined organic phases were dried $\left(\mathrm{MgSO}_{4}\right)$, the solvent was removed under reduced pressure yielding $\mathbf{5}$ quantitatively as brown oil. No purification was possible due to the instability of the product. $[\alpha]_{\mathrm{D}}{ }^{25}+20.2^{\circ}\left(c 0.82, \mathrm{CH}_{2} \mathrm{Cl}_{2}\right)\left\{\right.$ lit. $^{1}[\alpha]_{\mathrm{D}}{ }^{25}+18.5^{\mathrm{o}}(c$ 1, $\left.\left.\mathrm{CH}_{2} \mathrm{Cl}_{2}\right)\right\} ;{ }^{1} \mathrm{H}$ NMR $\left(300 \mathrm{MHz}, \mathrm{CDCl}_{3}\right) \delta 5.72(\mathrm{~d}, J=4.9 \mathrm{~Hz}, 1 \mathrm{H}), 5.25(\mathrm{~d}, J=4.9 \mathrm{~Hz}, 1 \mathrm{H}), 4.38-$ $4.28(\mathrm{~m}, 4 \mathrm{H}), 1.34(\mathrm{~m}, 6 \mathrm{H}) ;{ }^{13} \mathrm{C} \mathrm{NMR}\left(75 \mathrm{MHz}, \mathrm{CDCl}_{3}\right) \delta 166.58,166.52,79.97,79.34,63.2,14.1$, 14.0 .

Diethyl (2R, 3S)-3-azido-3-deoxy-tartrate (6). To a solution of 5 (3,2 g, $14.54 \mathrm{mmol})$ in DMF $(50 \mathrm{~mL})$ at r.t., sodium azide was added. The mixture was stirred at r.t. for $6 \mathrm{~h}$. After this time the solvent was evaporated under reduce pressure and the residue was dissolved in AcOEt (30 mL), washed with $\mathrm{NaCl}$ saturated $(3 \times 30 \mathrm{~mL})$ and dried with $\mathrm{Na}_{2} \mathrm{SO}_{4}(\mathrm{anh})$. After evaporation the residue was chromatographed (hexane/AcOEt, 3:1) yielding $6(2.1 \mathrm{~g}, 70 \%)$ as a palid oil. $[\alpha]_{\mathrm{D}}{ }^{25}+30.2(c$ 0.68, EtOH) $\left\{\right.$ lit. $^{1}[\alpha]_{\mathrm{D}}+32.8^{\circ}(c$ 1.64, EtOH $\left.)\right\} ;{ }^{1} \mathrm{H}$ NMR $\left(300 \mathrm{MHz}, \mathrm{CDCl}_{3}\right) \delta 4.62(\mathrm{~d}, J=5.4 \mathrm{~Hz}$, $1 \mathrm{H}), 4.21-4.35(\mathrm{~m}, 4 \mathrm{H}), 3.31(\mathrm{~d}, J=5.4 \mathrm{~Hz}, 1 \mathrm{H}), 1.30-1.28(\mathrm{~m}, 6 \mathrm{H}) ;{ }^{13} \mathrm{C} \mathrm{NMR}\left(75 \mathrm{MHz}, \mathrm{CDCl}_{3}\right) \delta$ 170.7, 166.9, 71.5 , 64.35, 62.6, 62.2, 13.96, 13.92. MS (EI) $m / z 232.6(\mathrm{M}+1)$.

3-Azido-3 deoxy-D-erythritol (7). At $0^{\circ} \mathrm{C}$ azide 6 (2.46 g, $\left.6.36 \mathrm{mmol}\right)$ was dissolved in EtOH (21 $\mathrm{mL})$ and sodium borohydride and lithium chloride were added (90 $\mathrm{mg})$. The reaction mixture was stirred at this temperature for $30 \mathrm{~min}$ and $6 \mathrm{~h}$ at r.t. After this time, a solution of $\mathrm{HCl}(1 \mathrm{M})$ was

\footnotetext{
${ }^{1}$ H.-S. Byun, L. He, R. Bittman, Tetrahedron, 2000, 56, 7051-7091.
} 
added at $0^{\circ} \mathrm{C}$ until $\mathrm{pH} 4$. The solvent was evaporated and the crude reaction was chromatographed (AcOEt/MeOH, 8:1) yielding 7 (589 mg, $63 \%)$. $[\alpha]_{\mathrm{D}}{ }^{25}-27.1$ (c 1, MeOH); ${ }^{1} \mathrm{H}$ NMR (300MHz, $\left.\mathrm{D}_{2} \mathrm{O}\right) \delta$ 3.8-3.7 (m, 1H), 3.6-3.4 (m, 5H); ${ }^{13} \mathrm{C}$ NMR (75MHz, $\left.\mathrm{D}_{2} \mathrm{O}\right) \delta$ 70.9, 64.7, 62.5, 61.1; MS (EI) $m / z$ 148.6 (M+1); Anal. Calcd for $\mathrm{C}_{4} \mathrm{H}_{9} \mathrm{~N}_{3} \mathrm{O}_{3}$ : C, 32.6; H, 6.1; N 28.5. Found: C 32.5; H 6.2; N 27.2.

3-Azido-2,4-O-benzylidene-3-deoxy-D-erythritol (8). To a solution of 7 (589 $\mathrm{mg}, 4.0 \mathrm{mmol}$ ) and benzaldehyde dimethylacetal $(1.8 \mathrm{~g}, 12 \mathrm{mmol})$ in anhydrous $\mathrm{MeCN}(30 \mathrm{~mL})$, p-TsOH was added $(20 \% \mathrm{~mol})$. The solution was then stirred under inert atmosphere for $32 \mathrm{~h}$. After this time solvent was evaporated under reduced pressure and the residue was dissolved in AcOEt $(20 \mathrm{~mL})$, washed with $\mathrm{NaCl}$ saturated $(3 \times 30 \mathrm{~mL})$ and dried with $\mathrm{Na}_{2} \mathrm{SO}_{4}(\mathrm{anh})$. After evaporation the crude reaction was chromatographed (hexane/AcOEt, 4:1) yielding 8 (423 mg, 45\%) as a yellow oil. $[\alpha]_{\mathrm{D}}{ }^{25}-28.8^{\mathrm{o}}\left(c\right.$ 0.5, $\left.\mathrm{CH}_{2} \mathrm{Cl}_{2}\right) ;{ }^{1} \mathrm{H}$ NMR $\left(300 \mathrm{MHz}, \mathrm{CDCl}_{3}\right) \delta$ 7.4-7.25 (m, 5H), $5.54(\mathrm{~s}, 1 \mathrm{H}), 4.46$ $(\mathrm{dd}, J=5.7 \mathrm{~Hz}, 1 \mathrm{H}), 4.0-3.6(\mathrm{~m}, 5 \mathrm{H}), 2.35(\mathrm{~s}, 1 \mathrm{H}) ;{ }^{13} \mathrm{C} \mathrm{NMR}\left(75 \mathrm{MHz}, \mathrm{CDCl}_{3}\right) \delta 137.2,129.6$, 128.6, 126.4, 101.5, 80.5, 68.9, 62.3, 52.8; MS(EI) $m / z 236.3(\mathrm{M}+1)$; Anal. Calcd for $\mathrm{C}_{11} \mathrm{H}_{13} \mathrm{~N}_{3} \mathrm{O}_{3}$ : C, 56.1; H, 5.5; N 17.8 . Found: C 56.5; H 5.2; N 17.2.

3-Azido-2,4-O-benzylidene-D-erythrose (3). At $0^{\circ} \mathrm{C}$ Trichloroisocyanuric acid $(80 \mathrm{mg}, 0.34$ mmol) was added to a solution of alcohol $8(70 \mathrm{mg}, 0.29 \mathrm{mmol})$ in anhydrous $\mathrm{CH}_{2} \mathrm{Cl}_{2}(1.5 \mathrm{~mL})$, followed by addition of TEMPO $\left(0.4 \mathrm{mg}, 2.9 \times 10^{-3}\right)$. Then, the mixture was warmed to room

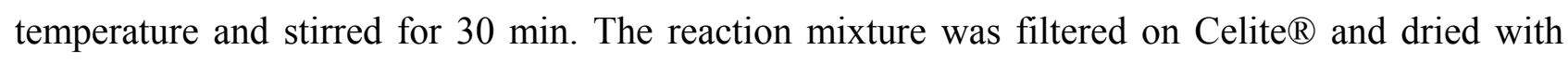
$\mathrm{Na}_{2} \mathrm{SO}_{4}$ (anh). After evaporation the residue was chromatographed (hexane/AcOEt, 4:1) yielding 3 (40 mg, 60\%) as a palid oil. $[\alpha]_{\mathrm{D}}{ }^{25}-7.4^{\mathrm{o}}\left(c \mathrm{0.6}, \mathrm{CH}_{2} \mathrm{Cl}_{2}\right) ;{ }^{1} \mathrm{H} \mathrm{NMR}\left(300 \mathrm{MHz}, \mathrm{CDCl}_{3}\right) \delta 7.7-7.4(\mathrm{~m}$, $5 \mathrm{H}), 5.54(\mathrm{~s}, 1 \mathrm{H}), 4.72-4.60(\mathrm{~d}, 1 \mathrm{H}), 4.6-4.39(\mathrm{~m}, 1 \mathrm{H}), 3.92-3.89(\mathrm{~m}, 1 \mathrm{H}), 3.75-3.62(\mathrm{~m}, 1 \mathrm{H}),{ }^{13} \mathrm{C}$ NMR $\left(75 \mathrm{MHz}, \mathrm{CDCl}_{3}\right) \delta 197.5,129.8,128.6,126.4,126.3,101.5,81.9,69.0,52.7$.

Diethyl (2S, 3S)-2,3-O-sulfinyl-tartrate (ent-5). $[\alpha]_{\mathrm{D}}^{25}-19.3^{\circ}\left(\mathrm{c} 1, \mathrm{CH}_{2} \mathrm{Cl}_{2}\right)$.

Diethyl (2S, 3R)-3-azido-3-deoxy-tartrate (ent-6). $[\alpha]_{\mathrm{D}}^{25}-32.2^{\mathbf{o}}(c 0.84$, EtOH).

3-Azido-3 deoxy-L-threitol (ent-7). $[\alpha]_{\mathrm{D}}^{25}+28.2$ (c 0.34, MeOH); MS (EI) $m / z 148.6(\mathrm{M}+1)$; Anal. Calcd for $\mathrm{C}_{4} \mathrm{H}_{9} \mathrm{~N}_{3} \mathrm{O}_{3}$ : C, 32.6; H, 6.1; N 28.5. Found: C 32.2; H 6.6; N 28.2. 
3-Azido-2,4-O-benzylidene-3-deoxy-L-threitol (ent-8). $[\alpha]_{\mathrm{D}}{ }^{25}+26.8^{\circ}\left(c 0.9, \mathrm{CH}_{2} \mathrm{Cl}_{2}\right)$; MS (EI) $m / z 236.3(\mathrm{M}+1)$; Anal. Calcd for $\mathrm{C}_{11} \mathrm{H}_{13} \mathrm{~N}_{3} \mathrm{O}_{3}$ : C, 56.1; H, 5.5; N 17.8 . Found: C 55.7; H 5.2; $\mathrm{N}$ 17.8.

3-Azido-2,4-O-benzylidene-L-threose (ent-3). $[\alpha]_{\mathrm{D}}{ }^{25}+8.6^{\circ}\left(c 1.6, \mathrm{CH}_{2} \mathrm{Cl}_{2}\right)$. 
<smiles>CC1(C)OCC(=O)[C@@H]([C@H](O)[C@H]2OC(c3ccccc3)OC[C@H]2N)O1</smiles>

- Key HMBC correlations of 9a:

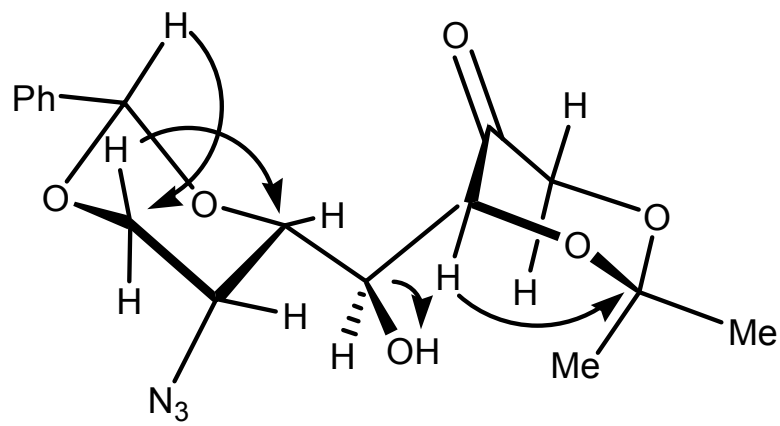

- Key NOESY correlations of 9a:

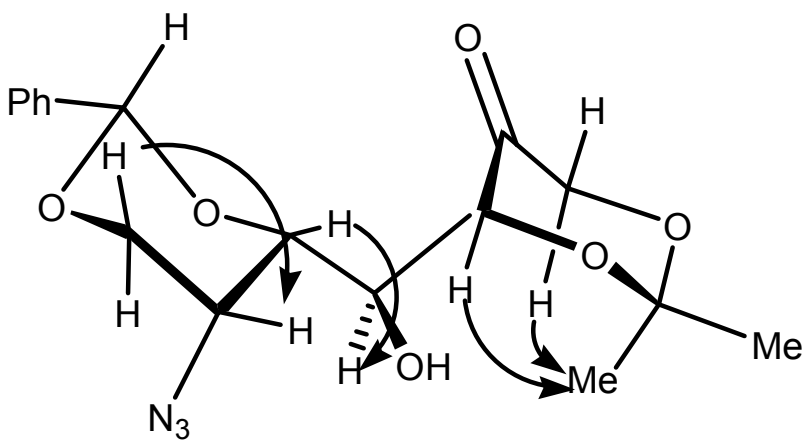




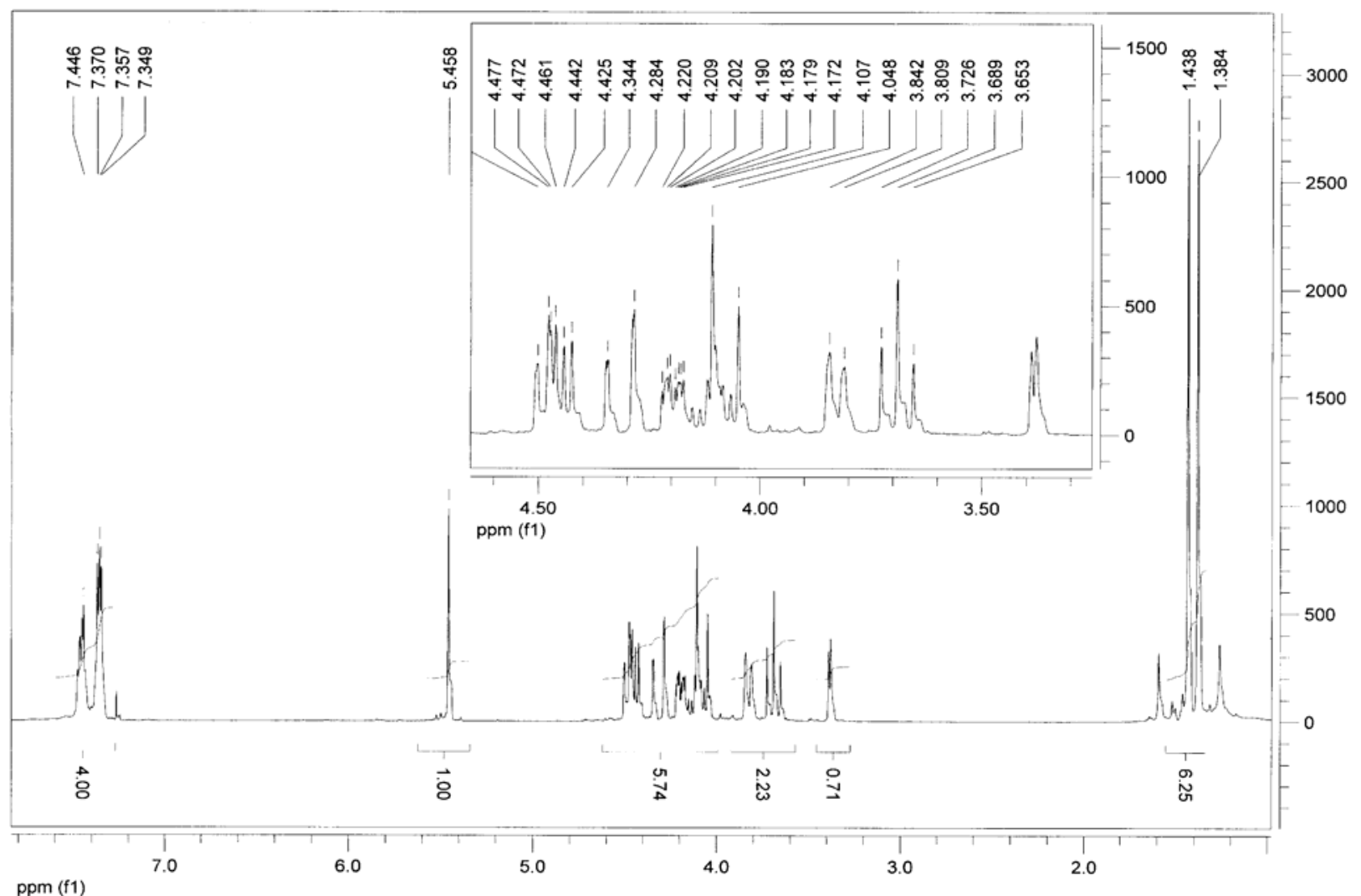

ppm (f1)

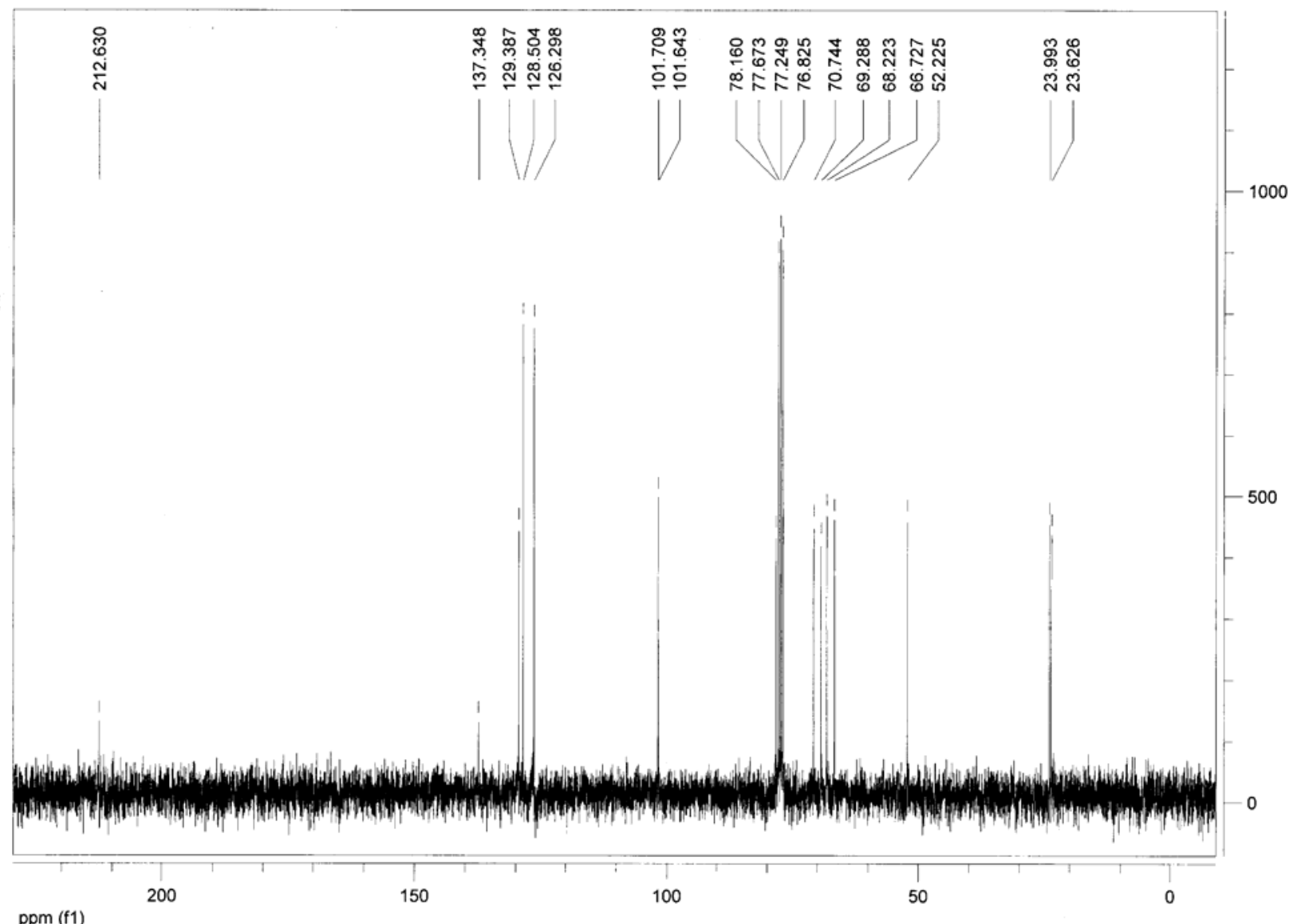


<smiles>CC1(C)OCC(=O)[C@@H]([C@H](O)C2OC(c3ccccc3)OC[C@H]2N)O1</smiles>

$9 b$

- Key COSY correlations of $\mathbf{9 b}$ :

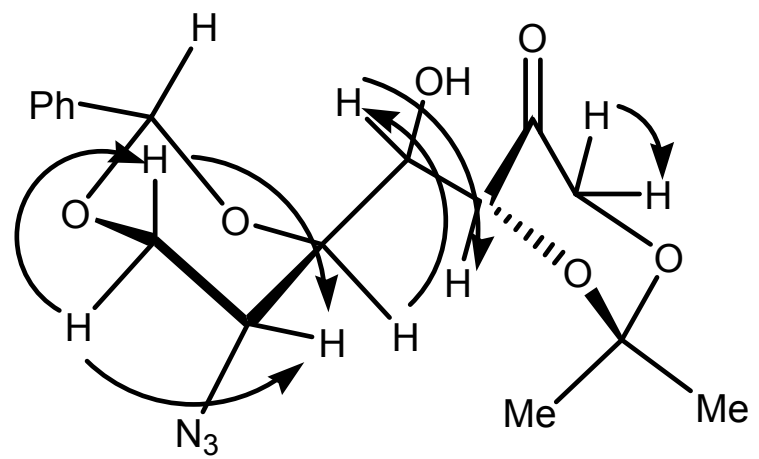

- Key HMBC correlations of $\mathbf{9 b}$ :

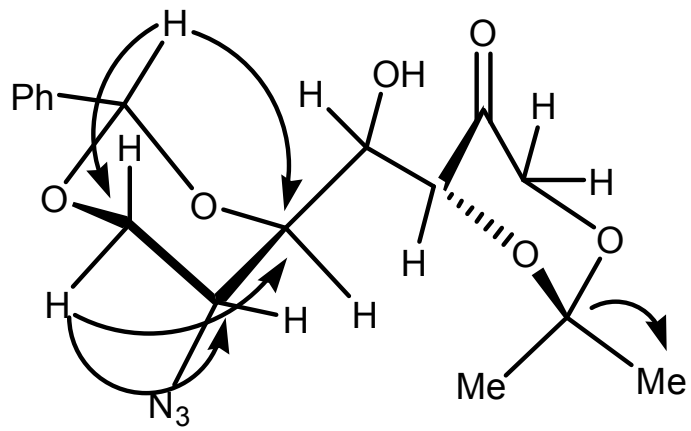

- Key NOESY correlations of $\mathbf{9 b}$

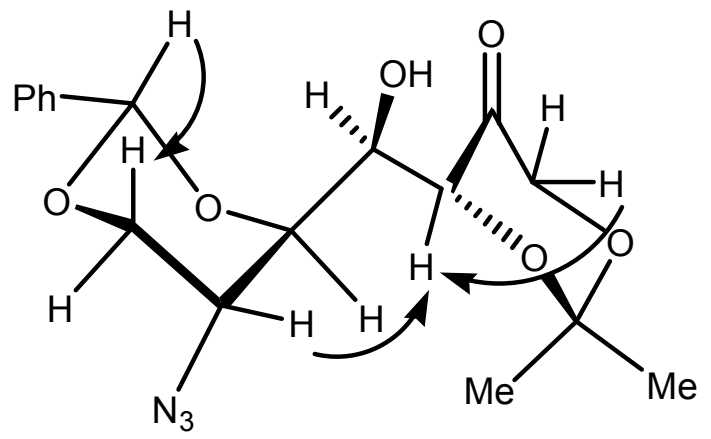




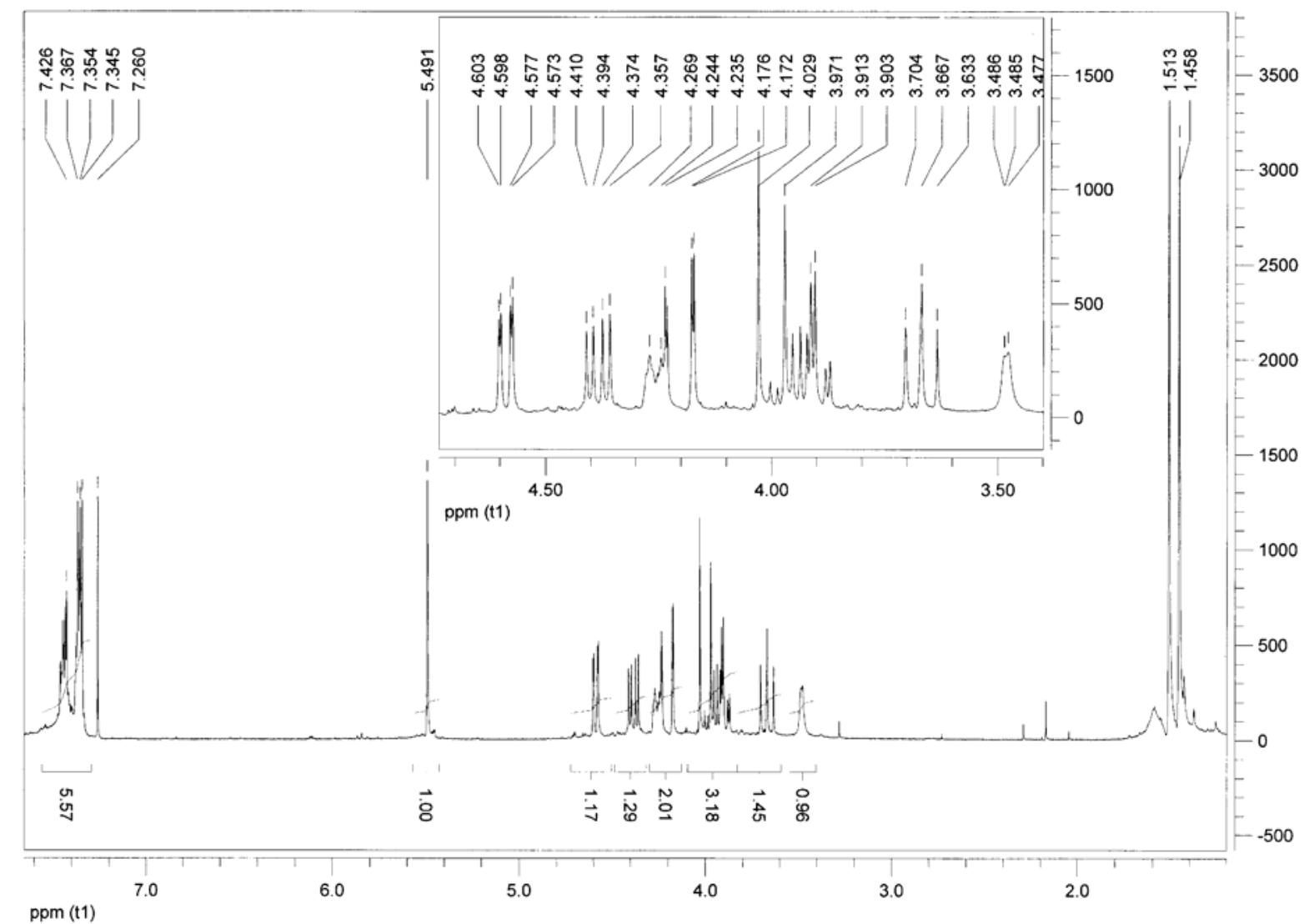

EC-C4-109-1

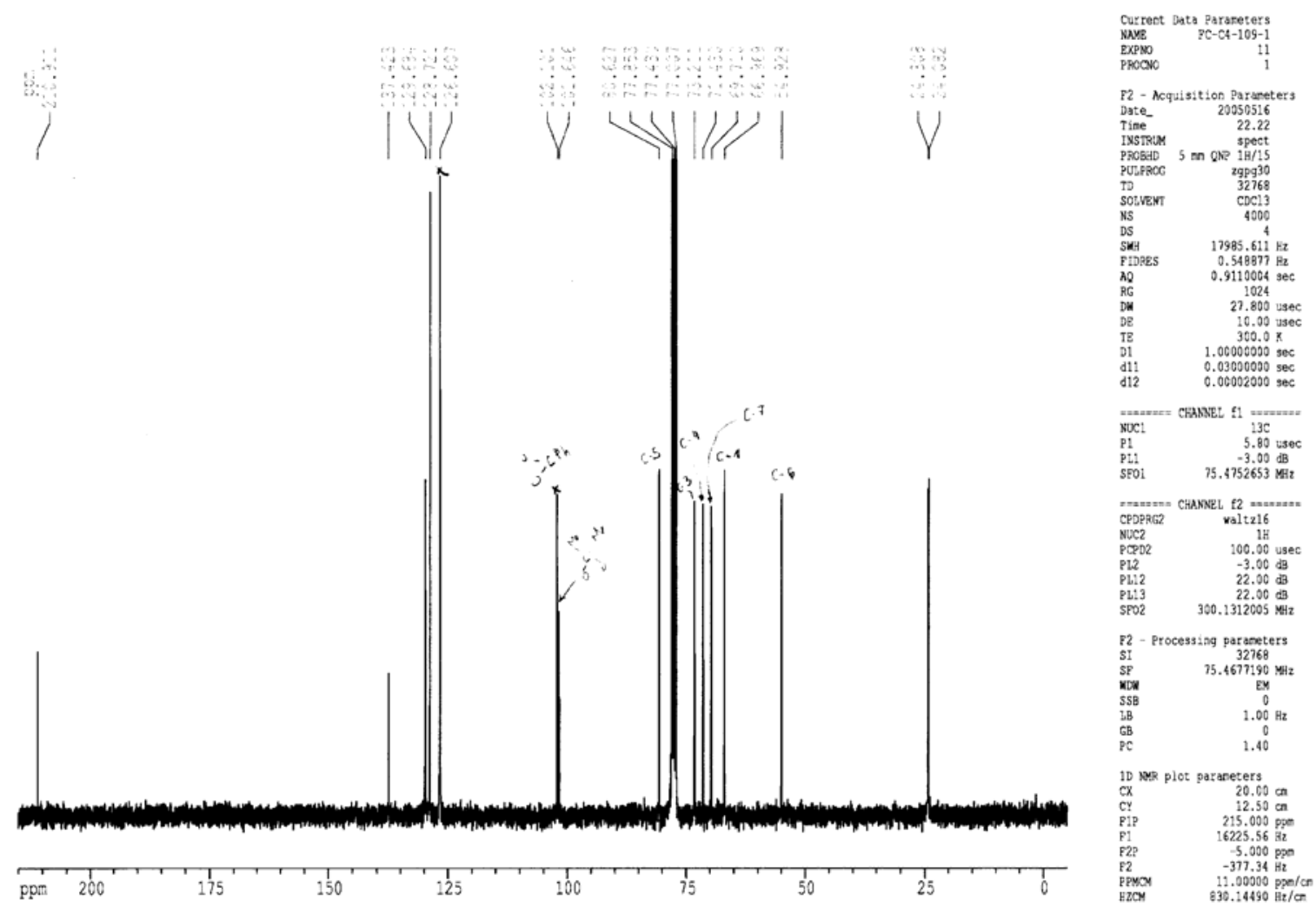


<smiles>CC(=O)[C@H](O)[C@H](O)[C@H]1OC(c2ccccc2)OC[C@H]1N</smiles>

10a

Relative configuration -anti was assigned by measurement of $J_{3,4}(13,3 \mathrm{~Hz})$ in compound 9a', obtained by acetalation of 9a with 2,2-dimethoxypropane in acetone.<smiles>CC(=O)C1OC(C)(C)O[C@H]1C1OC(c2ccccc2)OC[C@H]1N</smiles>

24

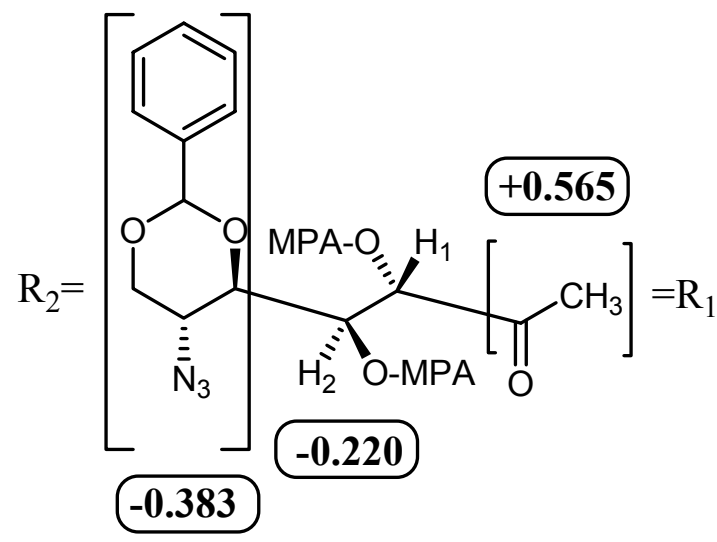

25

Absolute configuration was assigned by comparison of the $\Delta \delta^{\mathrm{RS}}$ signs of their ${ }^{1} \mathrm{H}$ NMR spectra of the corresponding diesters $(R)$ - and $(S)$ - methoxyphenylacetic acid (MPA). ${ }^{2}$ We have alredy applied this methodology for these kind of compounds ${ }^{3}$ showing that the absolute configuration for C-3 and C-4 can be obtained calculating the $\Delta \delta^{\mathrm{RS}}$ for R-1, R-2 and $\mathrm{H}-2$.

\footnotetext{
2 a) For a complete description of the theoric based: a)J. M. Seco, M. Marco, E. Quínoa, R. Riguera, Org. Lett. 2000, 21, 3261-3264. b) F. Freire, J. M. Seco, E. Quiñoá, R. Riguera, J. Org. Chem. 2005, 70, 3778-3790. c) General review of assignment by NMR: J. M. Seco, E. Quiñoá, R. Riguera, Chem. Rev. 2004, 104, 17-117.

3 F. Calderón, R. Fernández, F. Sánchez, A. Fernández-Mayoralas, Adv. Synth. Catal. 2005, 1395-1403
} 

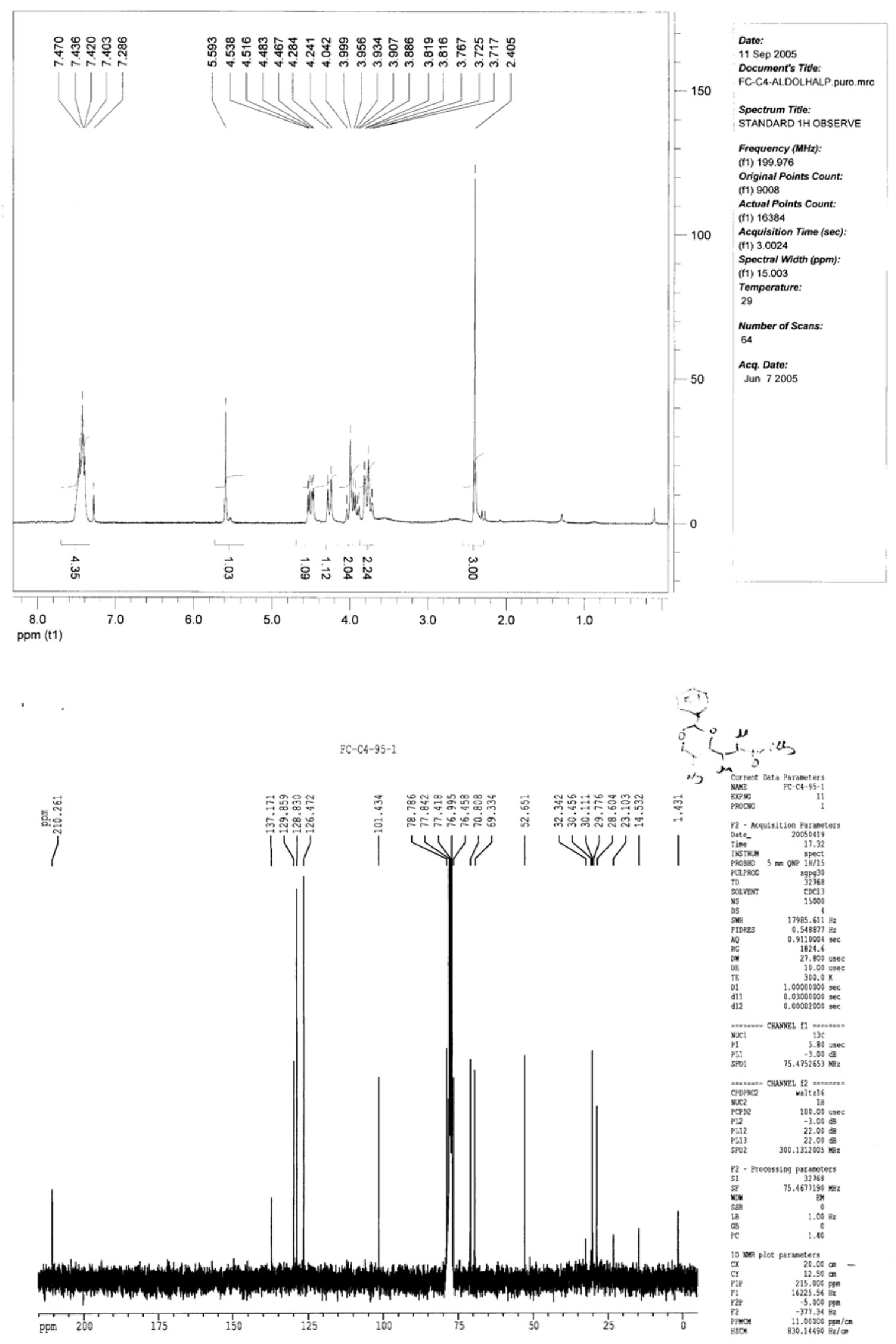
6-Azido-5,7-O-benzylidene-1,6-dideoxy-D-allo-hept-2-ulose (10b)<smiles>CC(=O)[C@H](O)[C@@H](O)[C@H]1OC([PH2+])OC[C@H]1N</smiles>

Crude NMR spectra
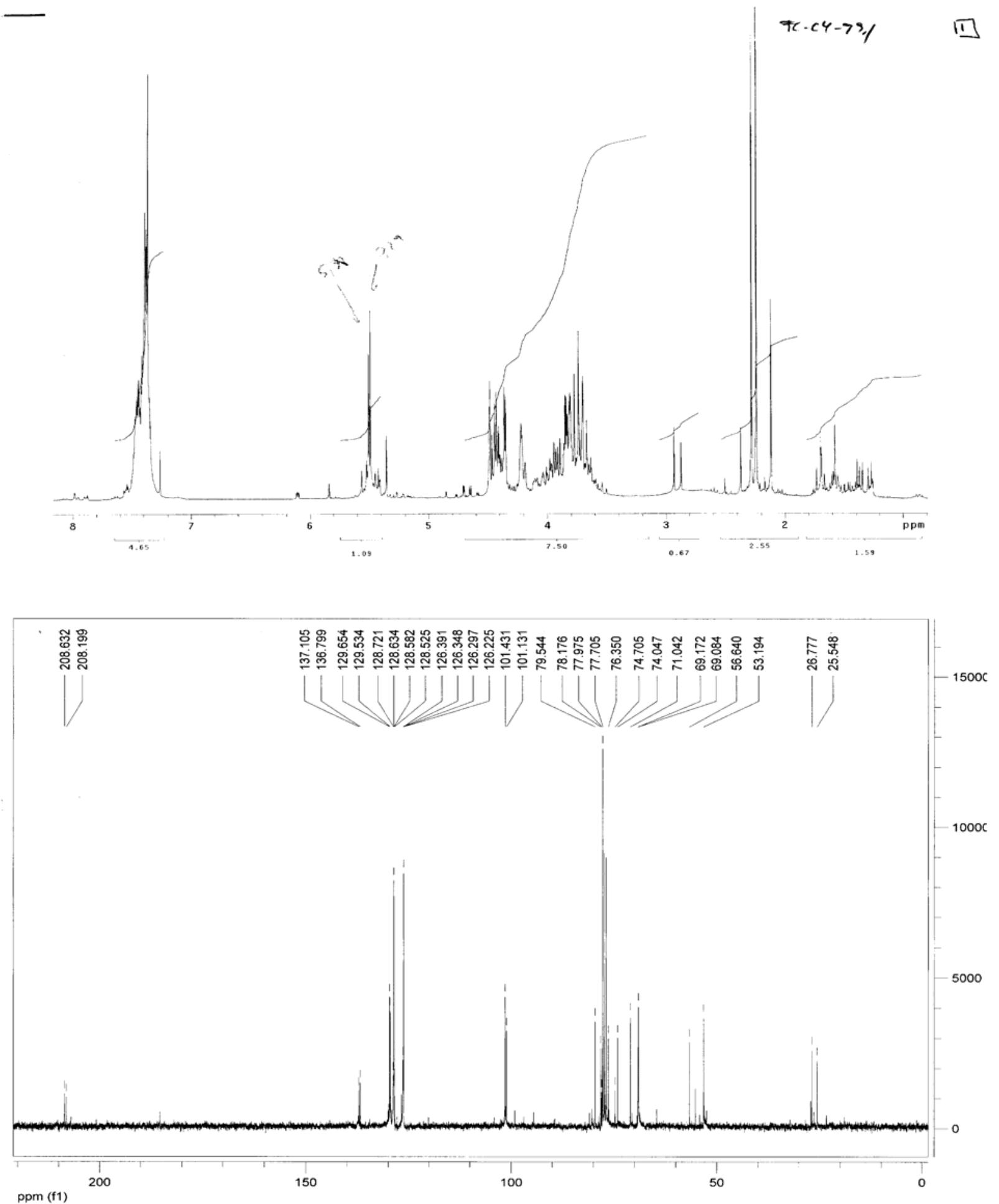
ß- Homomannojirimycin (11)

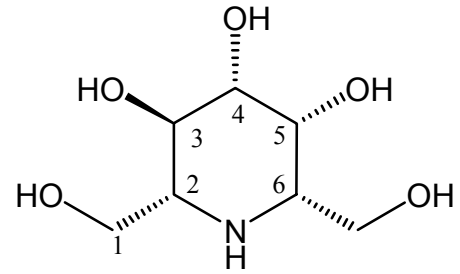

11

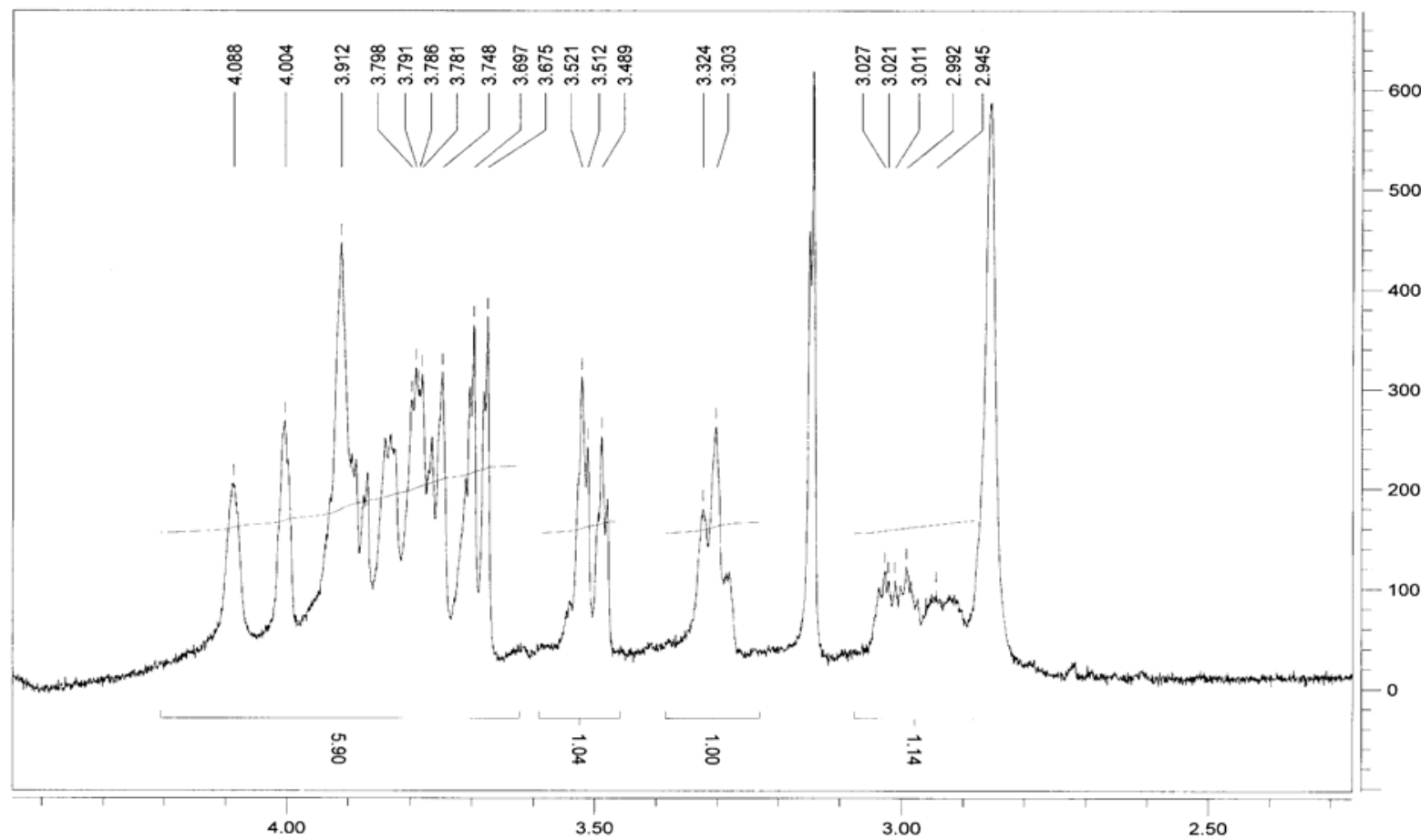

ppm (f1)

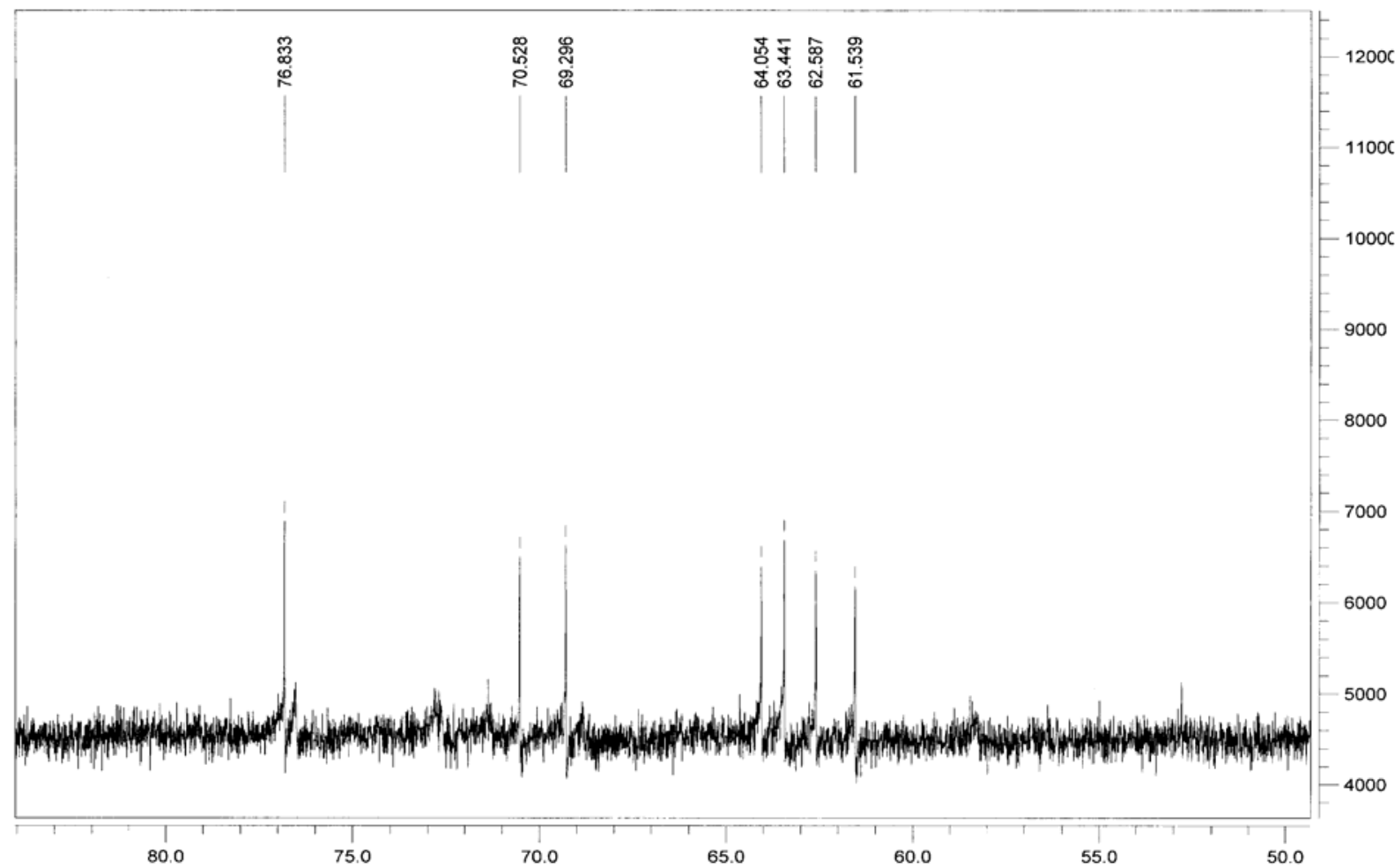

ppm (t1) 


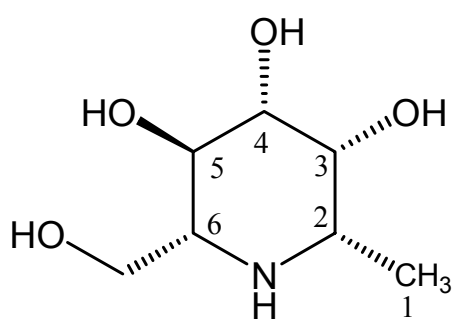

$13 a$<smiles>CC1N[C@H](CO)C(O)[C@H](O)[C@H]1O</smiles>

13b
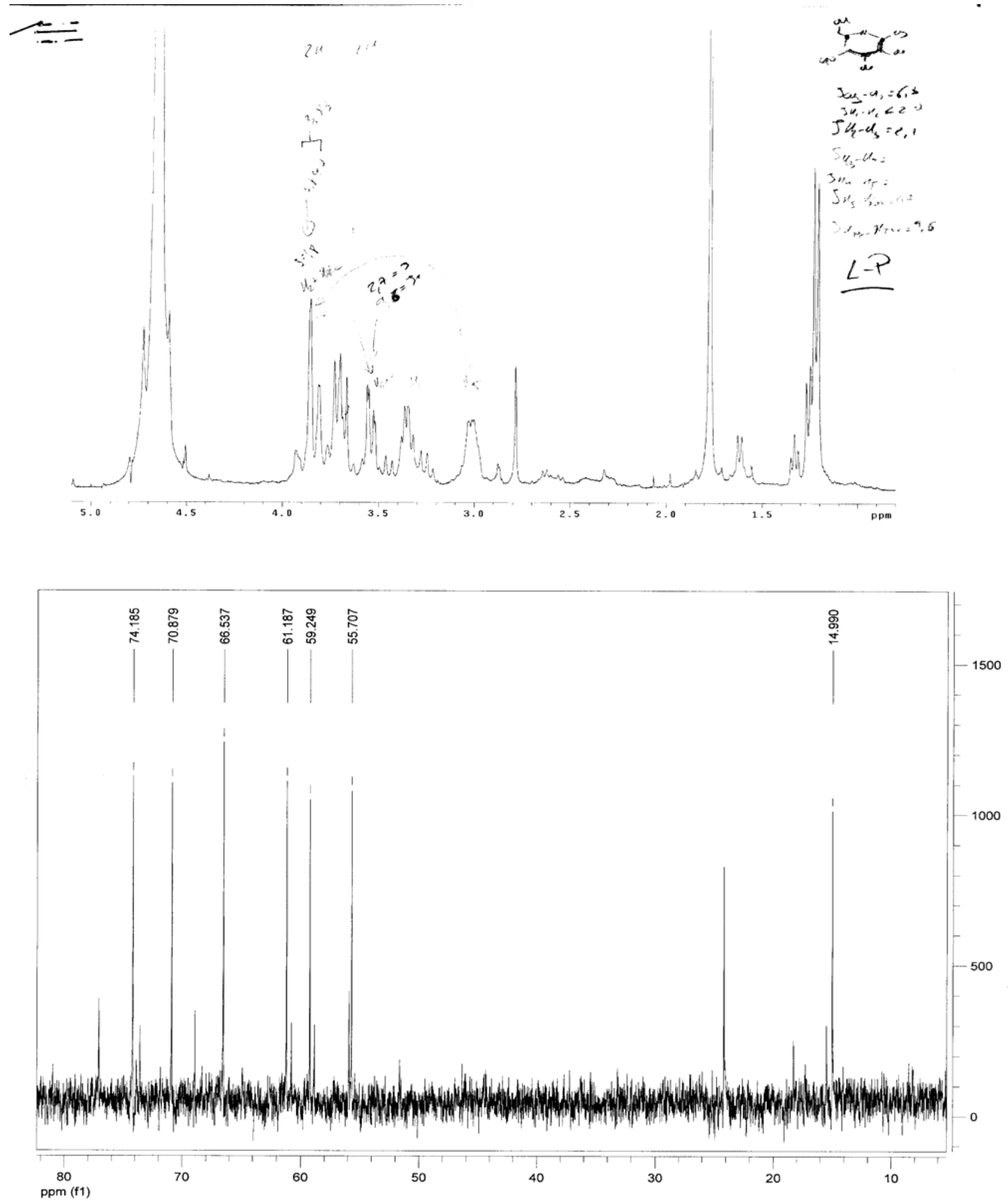


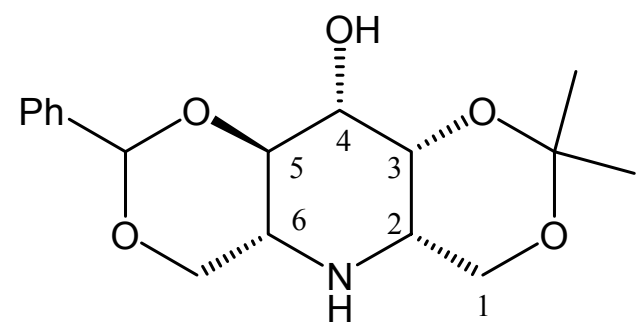

15

- Key COSY correlations for $\mathbf{1 5 .}$

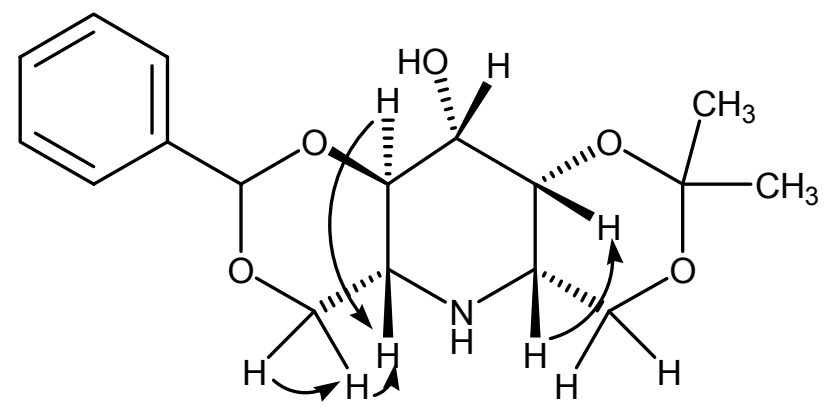

- Key HMBC correlations for $\mathbf{1 5 .}$

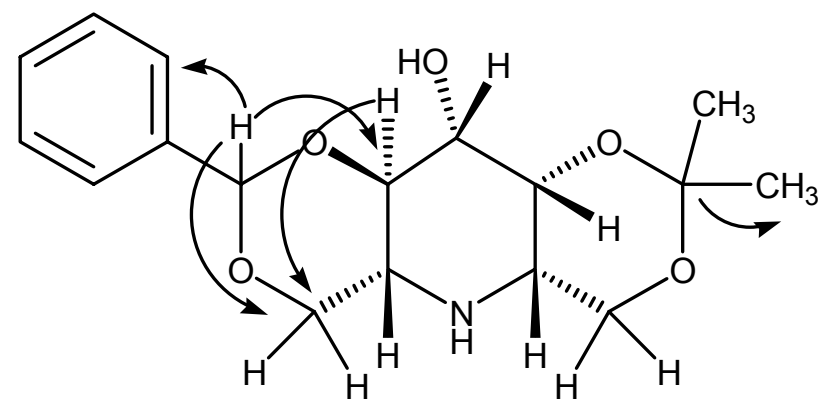

- Key NOESY correlations for $\mathbf{1 5 .}$

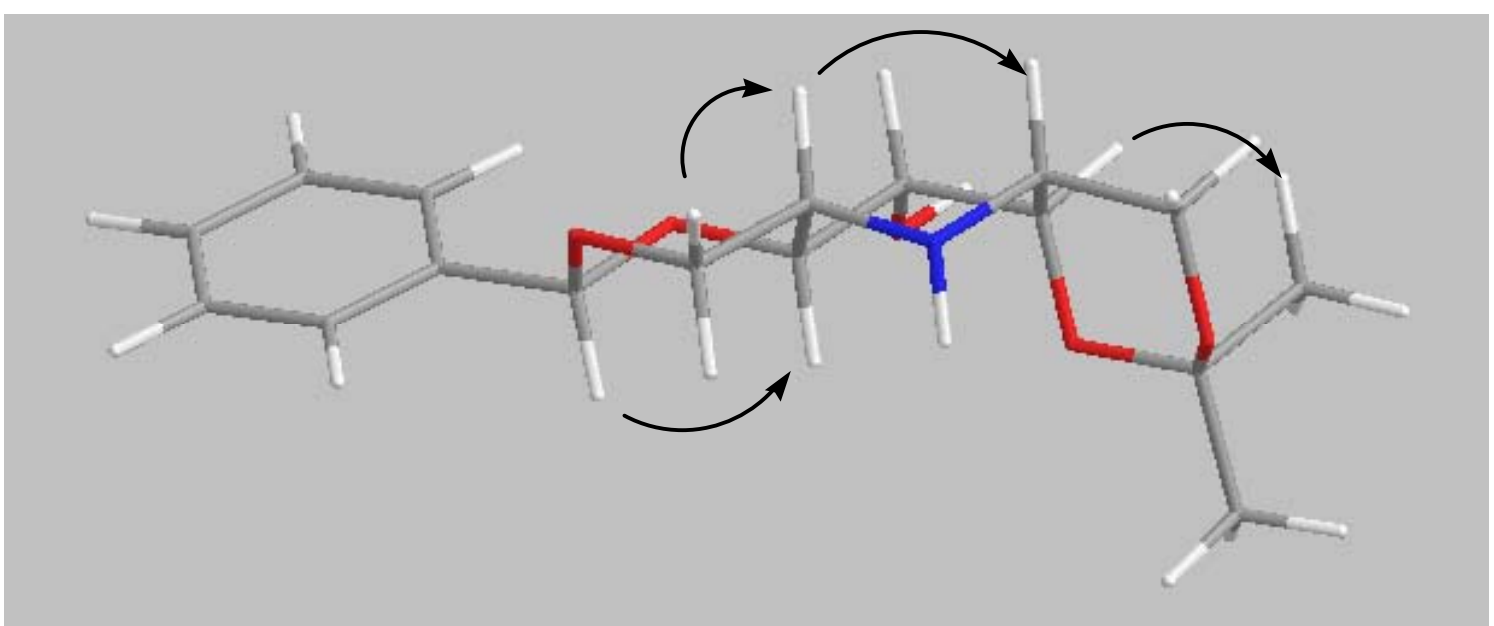



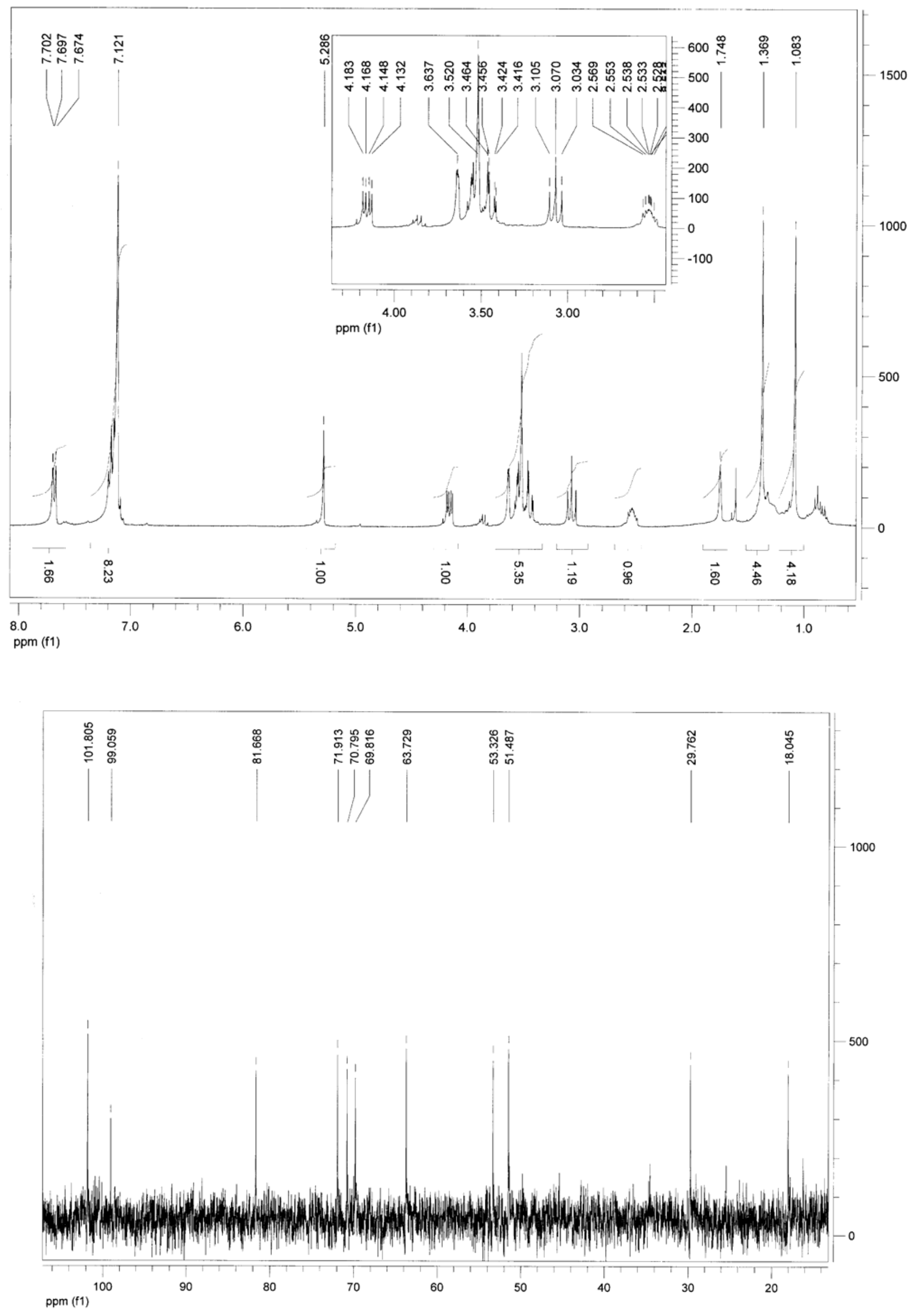


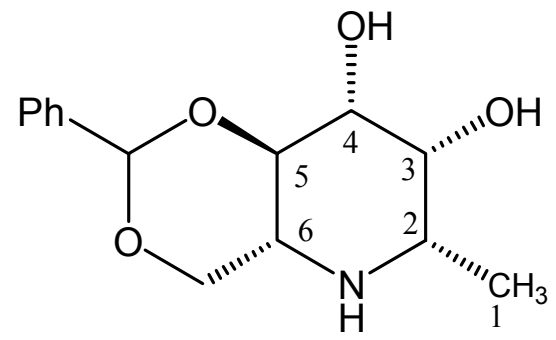

18 (major product)

- $\quad$ Key COSY correlations for $\mathbf{1 8 .}$

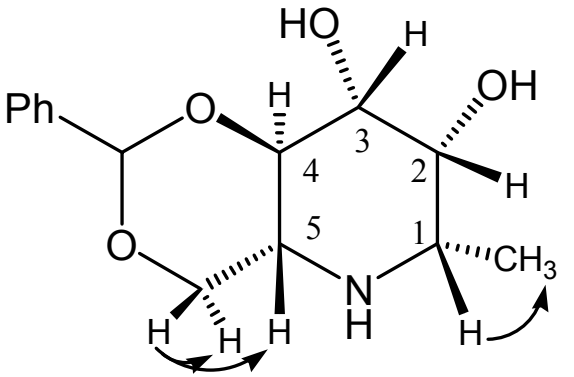

- Key HMBC correlations for $\mathbf{1 8}$.

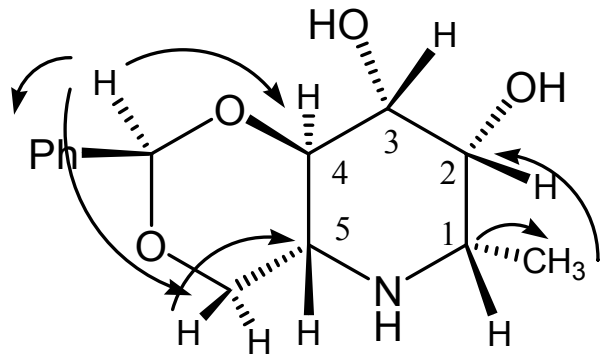

- Key NOESY correlations for 18.

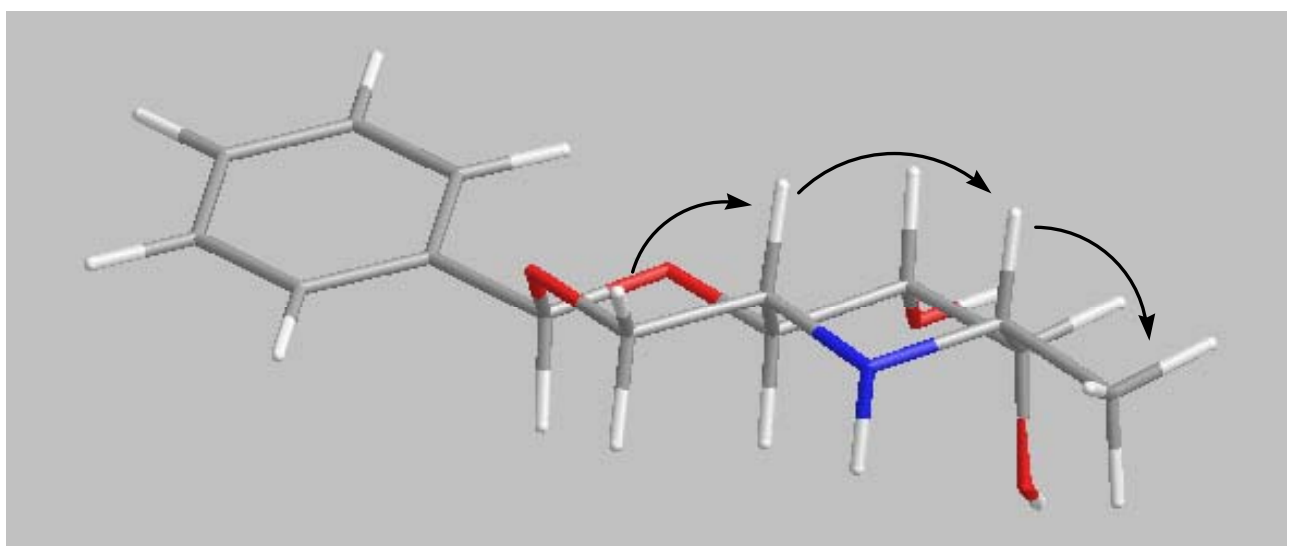




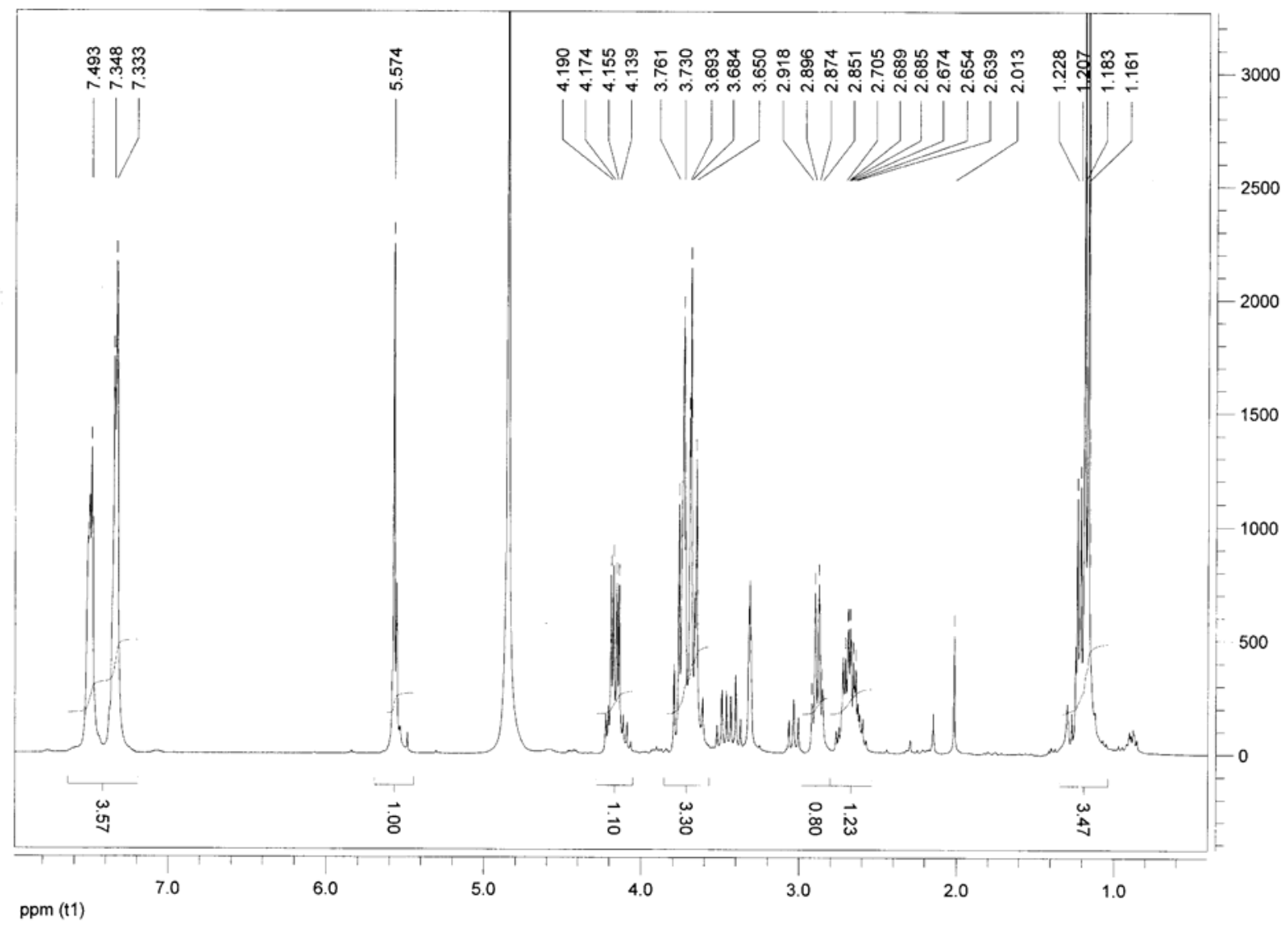

FC-C4-101-1

틈

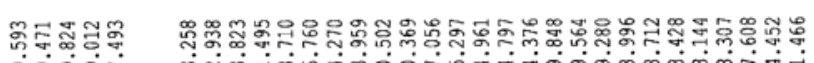

Carrent Dota Parareters
XRYE
EC-C4-101-1
EPROO
PROCNO

mi

F2 - Acquisit:on Parareters

$\begin{array}{lc}\text { Tate- } & 20050430 \\ \text { Tine } & 22.59 \\ \text { Trstary } & \text { spect }\end{array}$

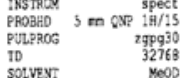

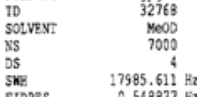

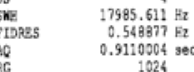

PG
JA
DE
TE

$\begin{array}{ll}0.1 .00000000 \mathrm{sec} \\ \text { di1 } & 0.0300000 \mathrm{sec}\end{array}$

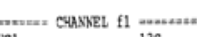

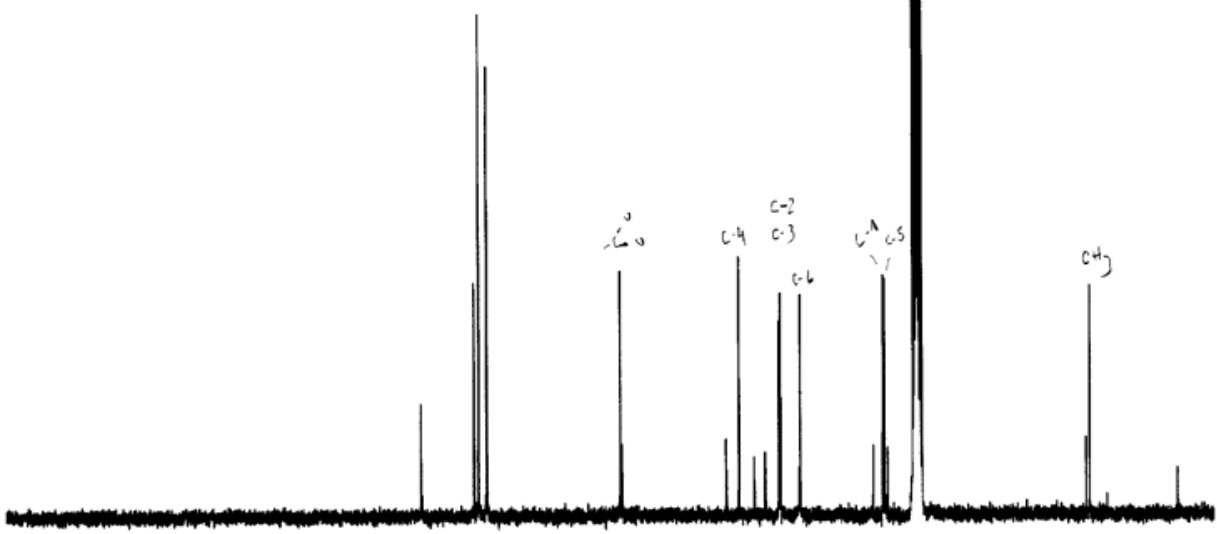

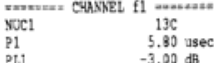

PEO1 $75.4752653 \mathrm{Miz}$

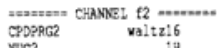

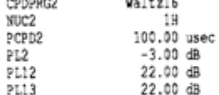

$3502 \quad 300.1312005 \mathrm{x}$

P2 - Processiog peraneters
S1
SF
SF

$\begin{array}{lc}\text { NDW } & \text { EM } \\ S S B & 0 \\ \text { LSB } & 1.00 \mathrm{HZ} \\ \text { IB } & .00 \\ P C & 0.10\end{array}$

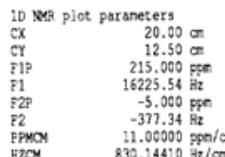

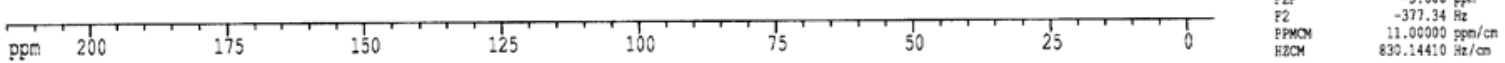

